\title{
Seasonal variation and predictors of intradialytic hypotension
}

\author{
Naoki Nakagawa ${ }^{1}$
}

Received: 27 July 2021 / Accepted: 1 August 2021 / Published online: 20 August 2021

(C) The Japanese Society of Hypertension 2021

Hypertension is an important public health issue because of its association with a number of significant diseases and adverse outcomes [1]. However, in addition to hypertension, intradialytic hypotension (IDH) is a major complication affecting a significant proportion of chronic hemodialysis patients and is associated with adverse longterm outcomes, including higher cardiovascular and allcause mortality [2]. It is estimated that approximately $20-30 \%$ of all dialysis sessions are affected by IDH [3]. IDH occurs due to the interaction among different factors, such as the ultrafiltration rate (UFR), cardiac output, and arteriolar tone. Therefore, excessive ultrafiltration may decrease cardiac output, especially when compensatory mechanisms (increase in heart rate, myocardial contractility, vascular tone, and redistribution of splanchnic blood flow) fail to be optimally recruited. The repeated disruption of end-organ perfusion by IDH may lead to various adverse clinical outcomes affecting the heart, central nervous system, kidneys, and gastrointestinal system. Potential interventions to decrease the incidence or severity of IDH include optimization of the dialysis prescription (cool dialysate, UFR adjustment, sodium profiling, and high-flux hemofiltration), interventions during the dialysis session (administration of midodrine and mannitol, food intake, intradialytic exercise, and intermittent pneumatic compression of the lower limbs) and interventions in the interdialytic period (reducing interdialytic weight gain and administration of blood pressure-lowering drugs). However, the evidence base is limited for many of these interventions, and optimal prevention and management of IDH await further clinical investigation. Just as seasonal variations in blood pressure (BP) are observed in the general population

Naoki Nakagawa

naka-nao@asahikawa-med.ac.jp

1 Division of Cardiology, Nephrology, Pulmonology and Neurology, Department of Internal Medicine, Asahikawa Medical University, Asahikawa, Japan
[4], predialysis systolic BP (SBP) in hemodialysis patients consistently follows a seasonal pattern as well, reaching a peak in the winter and a nadir in the summer [5]. This variation has been mostly attributed to seasonal changes in outside temperatures because high temperatures may result in vasodilatation with a decrease in peripheral vascular resistance, leading to a reduction in predialysis SBP. In contrast, nadirs in SBP are less affected by outside temperature and are more responsive to room temperature $[4,5]$. However, seasonal variations and predictors of intradialytic SBP decline are not well understood.

In the current issue of Hypertension Research, Uchiyama et al. [6] reported on an observational study conducted to demonstrate predictors and evaluate the seasonal variations in intradialytic SBP decline by analyzing the data obtained from a 1-year follow-up of 307 patients who had undergone 47,219 hemodialysis sessions. They assessed intradialytic SBP decline and assigned baseline or longitudinal clinical and laboratory parameters and environmental factors as covariates. In particular, they investigated the association of intradialytic SBP decline with the use of antihypertensive drugs and two important complications of patients undergoing hemodialysis, namely, chronic kidney diseasemineral and bone disorder (CKD-MBD) and malnutritioninflammation-atherosclerosis (MIA) syndrome [7, 8].

The decrease in the intradialytic SBP was the highest and lowest in the winter and summer, respectively, following a clear seasonal pattern. For CKD-MBD, a well-known extrarenal complication of $\mathrm{CKD}$, baseline and monthly serum phosphorus levels were positively correlated with SBP decline (estimate $1.55,95 \%$ confidence interval [CI], $0.30-2.80, P=0.02 ; 0.59,95 \%$ CI, 0.16-1.00, $P=0.007$ ), suggesting that higher serum phosphorus has short-, medium- and long-term detrimental effects on vascular calcification (VC), leading to increased arterial stiffness. Although the relationship between serum calcium or calcium intake and $\mathrm{VC}$ is not as well established as that of phosphorus, the positive correlation between monthly serum calcium level and intradialytic SBP decline seen in this study indicates 
Common causes of intradialytic hypotension

- Excessive ultrafiltration rate

- Reduced cardiac output

- Increased arteriolar tone

- Autonomic dysfunction

Possible approaches to prevent and treat

intradialytic hypotension by Uchiyama's study

- Calcium channel blocker use

- a blocker avoidance

- Serum phosphorus-lowering therapy

Fig. 1 Causes of intradialytic hypotension and approaches for its prevention and treatment

that calcium may have some immediate adverse effects on $\mathrm{VC}$ in hemodialysis patients.

MIA syndrome also occurs commonly and is one of the predictors of outcomes in hemodialysis patients [9]. In terms of MIA syndrome, C-reactive protein (CRP) decreases with increased dietary intake and serum albumin; however, this study showed that CRP also followed a seasonal pattern, similar to that of serum phosphorus, albumin, and normalized protein catabolism rate, with a peak in the winter and a nadir in the summer, suggesting that increased protein intake led to increased production of uremic toxins, which might result in systemic microinflammation [10]. Furthermore, excessive seasonal variation in intradialytic SBP decline might be correlated with higher comorbidity and poorer nutritional status in hemodialysis patients.

As many as $50-90 \%$ of end-stage renal disease patients suffer from hypertension [11], and finding the balance between managing hypertension and lowering the risk of IDH can be challenging. The mainstream practice is withholding BP-lowering medications prior to hemodialysis sessions since antihypertensive medications could theoretically exacerbate IDH. In statistical analyses conducted to determine the predictors of intradialytic SBP decline, increased use of $\alpha$ blockers was consistently associated with a greater intradialytic SBP decline $(3.25,95 \%$ CI, 1.53-4.97, $P<0.001)$, whereas calcium channel blocker $(\mathrm{CCB})$ use was associated with a smaller decline $(-4.58,95 \% \mathrm{CI},-5.84$ to $-3.33, P<$ 0.001 ) after adjustment for covariates with definite associations with IDH, including Charlson comorbidity index, predialysis SBP, UFR, and outside temperature. Furthermore, in a sensitivity analysis, the use of dihydropyridine CCBs was associated with a smaller decline in intradialytic SBP $(-6.32$, $95 \% \mathrm{CI},-9.12$ to $-3.52, P<0.001)$. Dihydropyridine CCBs are widely prescribed for the management of hypertension in Eastern Asians, as proposed by the Japanese guidelines on the management of hypertension [12], because they have a protective effect against target organ damage [13, 14] and cardiovascular events [15]. Furthermore, a recent study found that their use was associated with lower blood pressure variability than non-CCB therapy or no antihypertensive drugs [16]. Therefore, providers of dialysis care should implement pharmacological strategies such as withholding antihypertensive medications (especially $\alpha$ blockers) prior to dialysis and/or administering vasoconstrictor medications in an effort to reduce the frequency of IDH (Fig. 1).

In conclusion, the use of CCBs; appropriate control of CKD-MBD, especially serum phosphorus levels; and avoidance of $\alpha$ blockers and excessive protein intake might attenuate intradialytic SBP decline or variability that can lead to IDH. Further data are clearly needed to assess the clinical significance of the causal relationship of IDH with patient survival for optimal long-term management of patients undergoing hemodialysis.

\section{Compliance with ethical standards}

Conflict of interest The author declares no competing interests.

Publisher's note Springer Nature remains neutral with regard to jurisdictional claims in published maps and institutional affiliations.

\section{References}

1. Nakagawa N, Hasebe N. Impact of the 2017 American College of Cardiology/American Heart Association Blood Pressure Guidelines on the Next Blood Pressure Guidelines in Asia. Curr Hypertens Rep. 2019;21:2.

2. Kanno Y. Blood pressure management in patients receiving renal replacement therapy. Hypertens Res. 2021;44:7-12.

3. Palmer BF, Henrich WL. Recent advances in the prevention and management of intradialytic hypotension. J Am Soc Nephrol. 2008;19:8-11.

4. Parati G, Torlasco C, Pengo M, Bilo G, Ochoa JE. Blood pressure variability: its relevance for cardiovascular homeostasis and cardiovascular diseases. Hypertens Res. 2020;43:609-20.

5. Usvyat LA, Carter M, Thijssen S, Kooman JP, van der Sande FM, Zabetakis $\mathrm{P}$, et al. Seasonal variations in mortality, clinical, and laboratory parameters in hemodialysis patients: a 5-year cohort study. Clin J Am Soc Nephrol. 2012;7:108-15.

6. Uchiyama K, Shibagaki K, Yanai A, Kusahana E, Nakayama T, Morimoto K, et al. Seasonal variation and predictors of intradialytic blood pressure decline: a retrospective cohort study. Hypertens Res. 2021. https://doi.org/10.1038/s41440-021-00714-1.

7. Maruyama K, Nakagawa N, Saito E, Matsuki M, Takehara N, Akasaka K, et al. Malnutrition, renal dysfunction and left ventricular hypertrophy synergistically increase the long-term incidence of cardiovascular events. Hypertens Res. 2016;39:633-9.

8. Maruyama K, Nakagawa N, Koyama S, Maruyama JI, Hasebe N. Malnutrition increases the incidence of death, cardiovascular events, and infections in patients with stroke after rehabilitation. $\mathbf{J}$ Stroke Cerebrovasc Dis. 2018;27:716-23.

9. Nakagawa N, Matsuki M, Yao N, Hirayama T, Ishida H, Kikuchi K, et al. Impact of metabolic disturbances and malnutritioninflammation on 6-year mortality in japanese patients undergoing hemodialysis. Ther Apher Dial. 2015;19:30-9. 
10. Saijo Y, Utsugi M, Yoshioka E, Fukui T, Sata F, Nakagawa N, et al. Inflammation as a cardiovascular risk factor and pulse wave velocity as a marker of early-stage atherosclerosis in the Japanese population. Environ Health Prev Med. 2009;14:159-64.

11. Kanbay M, Ertuglu LA, Afsar B, Ozdogan E, Siriopol D, Covic A, et al. An update review of intradialytic hypotension: concept, risk factors, clinical implications and management. Clin Kidney J. 2020;13:981-93.

12. Umemura S, Arima H, Arima S, Asayama K, Dohi Y, Hirooka Y, et al. The Japanese Society of Hypertension Guidelines for the Management of Hypertension (JSH 2019). Hypertens Res. 2019;42:1235-481.

13. Klingbeil AU, Schneider M, Martus P, Messerli FH, Schmieder RE. A meta-analysis of the effects of treatment on left ventricular mass in essential hypertension. Am J Med. 2003;115:41-46.
14. Nakagawa N, Fujino T, Kabara M, Matsuki M, Chinda J, Kikuchi K, et al. Angiotensin II receptor blocker and long-acting calcium channel blocker combination therapy decreases urinary albumin excretion while maintaining glomerular filtration rate. Hypertens Res. 2011;34:1121-6.

15. Whelton PK, Carey RM, Aronow WS, Casey DE Jr., Collins KJ, Dennison Himmelfarb C, et al. 2017 ACC/AHA/AAPA/ABC/ ACPM/AGS/APhA/ASH/ASPC/NMA/PCNA Guideline for the Prevention, Detection, Evaluation, and Management of High Blood Pressure in Adults: A Report of the American College of Cardiology/American Heart Association Task Force on Clinical Practice Guidelines. Hypertension. 2018;71:e13-e115.

16. Liao R, Li J, Xiong Y, Lin L, Wang L, Sun S, et al. Association of peridialysis blood pressure and its variability with cardiovascular events in hemodialysis patients. Kidney Blood Press Res. 2018;43:1352-62. 